\title{
Comparaison pratique de méthodes de prévision de crue. Exemple de l'Aveyron
}

\author{
C. Thirriot, J.P. Bernède, H. Habaieb \\ Institut de mécanique des fluides \\ de l'Institut national polytechnique de Toulouse
}

\section{Introduction}

L'objectif du travail présenté ici a été la comparaison des possibilités de différentes méthodes simples de prévision des crues en temps réel. Le moyen a été la simulation numérique sur micro-ordinateur qui a permis de multiplier a bon marché les essais numériques (plus de 1500 essais ont été réalisés).

Nous avons choisi comme argument physique la rivière de l'Aveyron sur la suggestion du Service $\mathrm{Hy}$ drologique Centralisateur de Toulouse et nous avons bénéficié de l'aide cordiale et efficace de la Cellule Prévision et du Service Hydrologique de la Direction Départementale de l'Equipement du Tarn et Garonne de Montauban.

Bien sûr, nous sommes convaincus de l'importance d'une bonne connaissance physique du site et des phénomènes pour la prévision des crues, mais dans l'étude dont nous présenterons ici les résultats, nous avons travaillé en aveugle, appliquant des algorithmes de manière brutale sans paraître nous soucier des particularités géographiques. Ceci ne résulte pas d'un principe philosophique d'ignorer la nature, mais d'un parti pris occasionnel pour juger sans parti pris des performances de différents modèles suivant des critères simples et suffisamment généraux pour espérer que les conclusions qualitatives de cette expérience puissent être transposées à d'autres rivières.

A notre avis, une telle étude irréaliste mais systématique ne pouvait qu'être le fait d'universitaires, mais nous souhaitons qu'elle puisse aider les hydrologues de terrain à mieux cerner les promesses et les limites du concours de l'ordinateur dans la prévision des crues.

\section{Les méthodes}

Comme il s'agissait d'une remise en cause délibérée de tous les algorithmes habituellement utilisés pour le calcul des crues, nous avons voulu examiner aussi les performances de méthodes considérées comme triviales telles l'extrapolation et le lissage qui n'ont rien de spécifique aux crues. Dans notre attitude iconoclaste nous avons même tenté une expérience quasi irrationnelle, avec la mise en œuvre du SME que nous présenterons en détail plus loin.

Nous avons distingué les méthodes immédiates, qui n'utilisent que les informations à la station de prévision, des méthodes médiates ou à liaisons spatiales qui tiennent compte de l'information recueillie à l'extérieur de la station de prévision.

\section{Practical comparison of flood forecast methods. Example of the Aveyron River}

The main part of the studies consists of comparing the forecast flow rate values obtained by numerical simulation of the values actually assessed during the occurrence of about thirty floods on the River Aveyron. Deliberately, the foreccast algorithms tested are simple yet numerous. The comparison criteria used are the the standard deviation of values provided during a flood and the error on the maximum flow. An improvement is being sought according to three concepts: the use of the Kalman non-quantized filtering method, continuous competition of methods during floods and the mixing of result as regards several additional methods. Within the scope of this study and with the collaboration of hydrologist students and qualified forecasters, two forecast experiments have been made so as to be able to understand more of the mental mechanisms of weather forecasts with a view to setting up an expert system. 
Les algorithmes qui en sont déduits lient des valeurs de débit obtenues à partir de mesures limnimétriques, compte tenu des courbes de tarage supposées valables au moment de l'événement hydrologique considéré.

Pour toutes les méthodes, $t$ sera la date de la dernière observation et aussi le moment de la réalisation de la prévision à un délai $d$.

$\mathrm{La}$ fonction inconnue peut être le niveau ou le débit ou une fonction intermédiaire liée au débit (par exemple $\Phi=Q^{m}, Q$ étant lui-même lié à la hauteur d'eau par le barème de tarage approché par la relation $\left.Q=A\left(H-H_{0}\right)^{r}\right)$.

La fonction inconnue peut être encore l'accroissement pendant l'intervalle de temps $\Delta t$ d'une des grandeurs évoquées ci-dessus.

Cette dernière acception paraît particulièrement bien adaptée au problème de la prévision puisque à chaque instant où l'on fait la prévision, on dispose à partir de l'observation de la grandeur constituant comme la condition initiale.

\subsection{Les méthodes immédiates}

Elles présentent une formulation simple et s'obtiennent par projection suivant l'extrapolation ou la régression multiple.

\subsubsection{Les méthodes d'extrapolation}

L'extrapolation sera fondée tout simplement sur les procédés d'approximation mathématiques habituels à savoir l'interpolation et le lissage.

\section{a) L'extrapolation}

En supposant l'évolution polynomiale, en désignant par $\nabla$ l'opérateur de différence arrière, pour un polynôme de degré $p, \nabla^{p+1} Q=0$.

Par exemple pour l'extrapolation parabolique on obtient :

$$
Q(t+d)=3 Q(t)-3 Q(t-d)+Q(t-2 d)
$$

b) Le lissage

Nous avons proposé des algorithmes de lissage donnant plus de poids à la dernière valeur observée (THIRrior 1984) que nous avons appelés formules à pivot.

Par exemple pour un graphe linéaire passant obligatoirement par le dernier point et avec 3 points de base et double poids à l'origine, on obtient :

$$
\begin{aligned}
Q_{V}(t+d)=\left[16 . Q_{V}(t)+Q_{V}(t-d)\right. & \\
& \left.-6 . Q_{V}(t-2 d)\right] / 11
\end{aligned}
$$

\subsubsection{La régression multiple aux différences pre- mières}

Bien entendu, on peut appliquer les algorithmes précédents d'extrapolation et lissage aux différences premières. La régression est une forme de lissage généralisée mais pour la détermination des coefficients lorsque l'ordre de la régression n'est pas très élevé, on préfère utiliser les coefficients d'auto-corrélation que la méthode des moindres carrés.

Par exemple pour une régression multiple d'ordre 2

$$
\begin{aligned}
& y(t+d)=b_{1} y(t)+b_{2} y(t-\Delta t)+\varepsilon \\
& \text { avec } \quad d=m \Delta t \\
& \text { et } \quad b_{1}=\left(\rho_{m}-\rho_{1} \rho_{m+1}\right) /\left(1-\rho_{1}^{2}\right) \\
& b_{2}=\left(\rho_{m+1}-\rho_{1} \rho_{m}\right) /\left(1-\rho_{1}^{2}\right)
\end{aligned}
$$

Lorsque le délai de prévision est égal à l'intervalle de discrétisation de l'information, $m=1$.

\subsection{Méthodes à liaisons spatiales}

Ces méthodes tiennent compte de l'information recueillie à l'extérieur de la station de prévision. Cette information peut concerner les débits en une ou plusieurs stations et la pluie en un ou plusieurs postes.

Lors de l'étude systématique nous avons retenus les deux méthodes suivantes:

- méthode dérivée du modèle de Muskingum;

- méthode de régression multiple à deux stations.

Nous ferons le distinguo entre les modèles prenant en compte de manière explicite la pluie et ceux dans lesquels elle intervient de manière implicite en moyenne, dans le calage des coefficients.

\subsubsection{Modèles de la famille Muskingum}

Comme il s'agit d'un algorithme très classique chez les hydrologues, nous ne donnerons que la forme finale de l'algorithme que nous avons expérimenté avec pluie ou apport (ou retrait éventuellement).

Dorénavant les indices $m$ et $v$ repèrent les grandeurs respectivement amont et aval du bief.

$$
\begin{aligned}
Q_{V}(t+d)=a_{V 0} Q_{V}(t)+a_{m 0} Q_{m}(t) \\
+a_{m 1} Q_{m}(t+d)+\gamma S P\left(t-\tau^{\prime}\right)+\varepsilon(t)
\end{aligned}
$$

avec :

$\gamma \quad$ : coefficient de ruissellement.

$S$ : surface du sous bassin.

$P$ : pluie moyenne en espace et temps.

$\tau^{\prime} \quad$ : temps de concentration à l'exutoire.

Mais là encore l'alternative est possible :

- ou bien on suppose qu'en l'absence de pluie sur le sous bassin intermédiaire, il n'y a pas d'apport sensible et on doit utiliser la contrainte du régime permanent : $a_{V 0}+a_{m 0}+a_{m 1}=1$;

- ou bien on laisse flotter tous les coefficients ce qui signifie qu'une partie des apports est intégrée dans les termes de débit amont ou de débit aval à l'instant $t$ mais alors en niant l'existance physique du temps de concentration.

Tout ceci étant fait sur l'ajustement d'une crue antérieure. Le critère adopté est la minimisation de l'écart quadratique en essayant différentes valeurs de $\tau$ '.

\subsubsection{Modèle à régression multiple}

Ici encore nous donnerons directement l'algorithme avec pluie, avec deux observations antérieures aval pour tenir compte de la tendance (comme dans l'extrapolation) et avec une observation antérieure amont décalée du temps de propagation $t_{p}$ sur le bief. 


$$
\begin{aligned}
Q_{V}(t+d)= & a_{m 0} Q_{m}\left(t+d-t_{p}\right)+a_{V 0} Q_{V}(t) \\
& +a_{V 1} Q_{V}(t-d)+a_{p 0} S P\left(t-t_{c}\right)+\varepsilon(t)
\end{aligned}
$$

Nous avons aussi utilisé trois observations antérieures de débit amont pour transposer la méthode de Bachet, qui fait intervenir la courbure pour apprécier l'amortissement sur le bief.

\section{Critères de comparaison}

Le premier critère de la prévision est l'écart type des écarts entre prévision $Q_{p}$ et observation $Q_{\text {obs. }}$.

$$
\varepsilon_{Q}^{2}=\sum_{j=1}^{N}\left(Q_{p}(j \Delta t)-Q_{\text {obs }}(j \Delta t)\right)^{2} / N
$$

avec $N$ : nombre total d'observations au cours de la même crue.

En fait dans le but de pouvoir comparer les méthodes pour des crues différentes, nous avons considéré l'erreur relative obtenue par comparaison à la valeur maximale

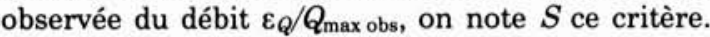

Mais pour les applications de la prévision, un fort écart sur la valeur maximale du débit présente plus de dommage que sur les valeurs des petits débits en début ou en fin de crue. Ainsi nous proposons l'écart relatif $\varepsilon_{Q_{\max }} / Q_{\max \text { obs. }}$. Ce sera notre deuxième critère d'évaluation noté $S^{\prime}$.

\section{Les résultats}

Sur la rivière de l'Aveyron nous avons pu recueillir 31 crues sur la période 1974-1984 (tableau 1) à 6 stations

\begin{tabular}{|c|c|c|c|c|c|}
\hline Crue & pointe & $\begin{array}{l}Q \operatorname{lan} x \\
=1 / 5\end{array}$ & (b) & $\begin{array}{c}\text { Duree } \\
\text { (b) }\end{array}$ & $10^{2}=$ \\
\hline $01 / 02-12 / 02 / 74$ & 2 & 224 & 156 & 288 & .70 \\
\hline $15 / 02-26 / 02 / 74$ & 1 & 87 & 54 & 288 & 10 \\
\hline $16 / 01-22 / 01 / 75$ & 1 & 67 & 72 & 168 & 23 \\
\hline $27 / 01-05 / 02 / 75$ & 1 & 80 & 66 & 240 & 36 \\
\hline $30 / 11-14 / 12 / 76$ & 1 & 118 & 86 & 360 & 70 \\
\hline $03 / 05-11 / 05 / 77$ & 1 & 76 & 60 & 216 & 27 \\
\hline $18 / 05-23 / 05 / 77$ & 2 & 64 & 50 & 144 & 20 \\
\hline $25 / 08-05 / 09 / 77$ & 1 & 88 & 44 & 280 & 23 \\
\hline $23 / 01-31 / 01 / 78$ & $i$ & 125 & 64 & 210 & 44 \\
\hline $01 / 02-14 / 02 / 78$ & $i$ & 125 & 72 & 336 & 57 \\
\hline $16 / 02-28 / 02 / 78$ & 1 & 110 & 10 & 312 & 57 \\
\hline $10 / 01-15 / 01 / 79$ & 1 & 81 & 20 & 124 & 20 \\
\hline $14 / 03-19 / 03 / 79$ & $i$ & 84 & 28 & 124 & 22 \\
\hline $25 / 10-31 / 10 / 79$ & 1 & 122 & 56 & 168 & 25 \\
\hline $15 / 12-23 / 12 / 79$ & 1 & 121 & 54 & 216. & 44 \\
\hline $28 / 12-04 / 01 / 80$ & 1 & 155 & 86 & 192 & 46 \\
\hline $05 / 01-12 / 01 / 80$ & 1 & 68 & 36 & 192 & 27 \\
\hline $22 / 01-31 / 01 / 80$ & 2 & 66 & 82 & 216 & 32 \\
\hline $23 / 02-29 / 02 / 80$ & 1 & 104 & 16 & 148 & 19 \\
\hline $03 / 01-12 / 01 / 81$ & 1 & 97 & 54 & 240 & 39 \\
\hline $15 / 01-27 / 01 / 81$ & 3 & 120 & 150 & 312 & 72 \\
\hline $29 / 03-05 / 04 / 81$ & 3 & 82 & 104 & 180 & 35 \\
\hline $10 / 05-19 / 05 / 8$ ! & 1 & 129 & 74 & 240 & 52 \\
\hline $12 / 12-20 / 12 / 81$ & 1 & 158 & 54 & 216 & 50 \\
\hline $06 / 01-14 / 01 / 82$ & 1 & 115 & 48 & 216 & 40 \\
\hline $06 / 12-10 / 12 / 82$ & 1 & 80 & 46 & 108 & 18 \\
\hline $10 / 12-27 / 12 / 82$ & 1 & 158 & 76 & 400 & 110 \\
\hline $25 / 02-06 / 03 / 83$ & 2 & 119 & 64 & 240 & 53 \\
\hline $25 / 04-03 / 05 / 83$ & 2 & 125 & 70 & 216 & 43 \\
\hline $21 / 01-28 / 01 / 84$ & 1 & 125 & 98 & 192 & 42 \\
\hline $07 / 02-14 / 02 / 84$ & $i$ & 133 & 54 & 192 & 49 \\
\hline
\end{tabular}

Tableau 1. - Caractéristiques des crues de Villefranche.

Alt1tude du zoro: 260 sGF $\triangle S=S u-S_{0}=1000-560=440 \mathrm{~km}$ hydrométriques, sur le cours d'eau principal de l'amont vers l'aval : Onet le Château, Villefranche de Rouergue, Laguépie 1, Négrepelisse et Loubéjac, sur l'affluent du Viaur : Laguépie 2 et les informations pluviométriques à deux stations : Rodez et Négrepelisse.

Les essais présentés ici portent sur le bief Onet le Château (Rodez), Villefranche de Rouergue encore situé dans le bassin supérieur, cas apparemment intermédiaire pour les crues, entre la relation pluie-débit et le phénomène de propagation.

\subsection{Présentation de quelques résultats synthé- tiques}

Dans ce qui suit pour les méthodes nécessitant un calage des coefficients, nous avons utilisé pour l'ajustement la crue précédente de même saison.

Pour rassembler les résultats d'essai de prévisions de manière synthétique nous avons regroupé pour une même crue sur une planche les indications des erreurs dues aux différentes méthodes de prévision et pour différents délais de prévision (figures 1 et 2).

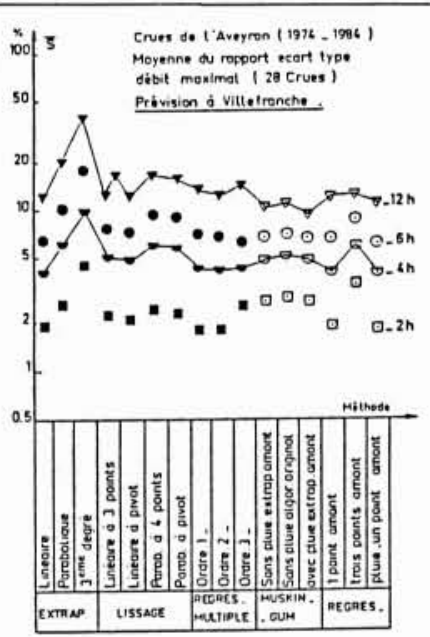

1. Moyenne du rapport écart type/débit maximal. Prévision à Villefranche.

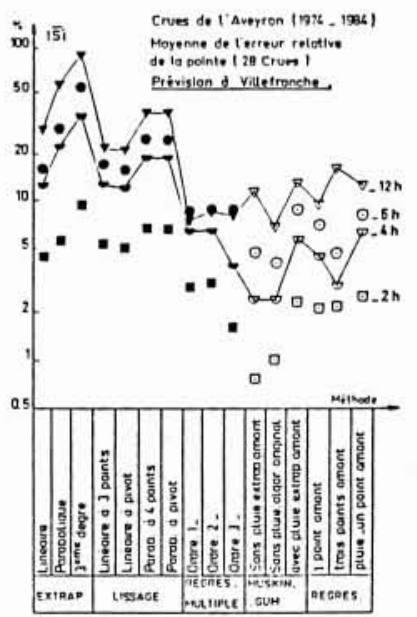

2. Moyenne de l'erreur relative de la pointe. Prévision à Villefranche. 


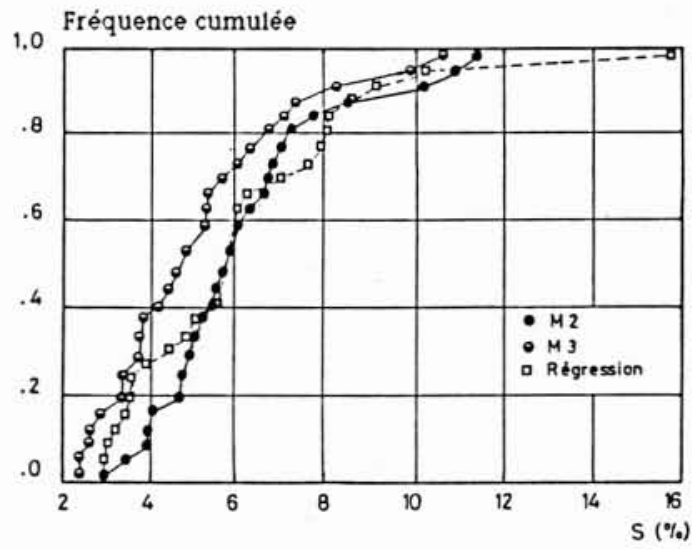

3. Fonction de répartition des écarts $S(d=6 h)$.

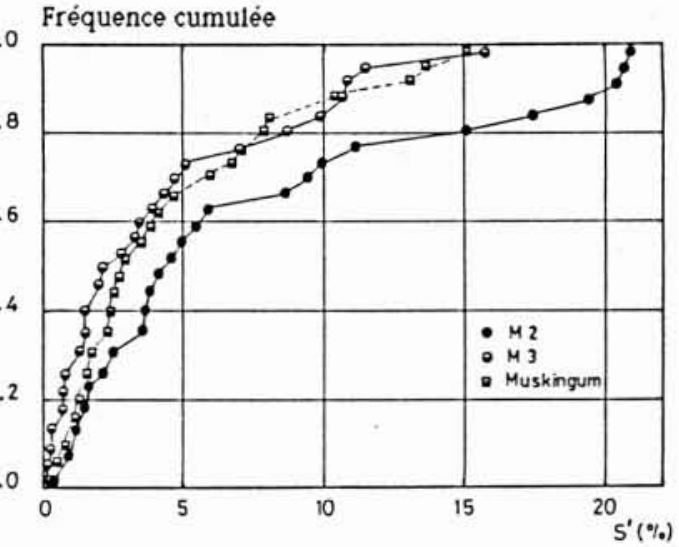

4. Fonction de répartition des erreurs $S^{\prime}(d=6 h)$.
D'autre part nous avons construit les graphes de répartition des erreurs moyennes (critère $S$ ), et des erreurs sur le débit maximal (critère $S^{\prime}$ ); ceci pour chaque méthode.

Quelles sont les conclusions essentielles qui ressortent du simple examen de ces figures?

1) Du point de vue extrapolation et lissage, les algorithmes les plus simples sont les plus satisfaisants. Pas besoin de sophistiquer les formules, les projections linéaires sont les meilleures et ce paradoxalement, d'autant plus que le délai de prévision augmente.

2) En dépit de toutes les critiques théoriques qu'on peut lui adresser, la méthode de Muskingum est la plus robuste, elle résiste bien à l'augmentation du délai de prévision surtout pour l'appréciation du débit maximal.

3) Pour le procédé de régression, prendre trois informations à l'amont ne paraît pas améliorer la prévision de manière décisive.

4) Considérer l'information pluviométrique amène un mieux surtout pour la méthode de régression avec un point amont, mais le gain reste fragile par rapport aux procédés sans prise en compte explicite de la pluie (ceci vraisemblablement à cause de la forte corrélation existant entre la pluie et le débit, et prise en compte de manière implicite dans la détermination des coefficients sur les crues antérieures).

5) La prise en compte des différences premières ne bouleverse pas les conclusions concernant l'écart quadratique moyen mais amène un mieux sensible pour ce qui concerne la prévision du débit maximal pour les pas de temps élevés. Ceci est important du point de vue de la sécurité.

\subsection{Traitement des erreurs}

L'étude des fonctions d'autocorrélation des écarts entre prévision et observation nous a montré que les corrélogrammes présentent tous à peu près la même forme et que leur allure ne dépend ni de la qualité de la prévision ni du délai fixé.

L'allure des corrélogrammes n'autorisant pas l'utilisation de modèles de correction autorégressifs, il nous a fallu chercher des méthodes spécifiques de correction de la prévision elle-même, ou de correction des paramètres. Mais il semble difficile par le traitement des erreurs d'améliorer, au sens tout à la fois des critères $S$ et $S^{\prime}$, les résultats à toutes les étapes d'évolution et de manière significative. Il y aura lieu de distinguer la montée, l'étale et la décrue.

\subsection{Prise en compte de la saison et d'un indice d'humidité}

A partir des 31 crues dont nous disposions, nous avons examiné l'influence, sur la valeur des coefficients des algorithmes, de la saison et d'un indice pondéré évanescent des pluies antérieures. Il y a peu de changement sur l'estimation de $S$ mais une amélioration sensible sur $S^{\prime}$ (détermination du maximum) par rapport à la méthode de la crue saisonnière précédente.

\section{Compétition des méthodes}

Dans le cours de cette étude se dessine peu à peu l'idée que chaque méthode a ses avantages et ses inconvénients. Dès 1984 l'un de nous (THIRrIơ 1984) avait proposé de maintenir en concurrence plusieurs méthodes tout au long de la prévision. Nous avons pensé à deux approches possibles:

a) La compétition pure et simple suivant la valeur pondéré des écarts absolus $e^{\star}$ aux temps récents :

$$
e^{\star}=1 / 2 \cdot|e(t)|+1 / 3 \cdot|e(t-d)|+1 / 6 \cdot|e(t-2 d)|
$$

b) Le panachage en association par pondération des résultats obtenus, compte tenu que certaines méthodes sousestiment les débits alors que d'autres les surestiment.

Après analyse (au sens du risque Bayésien) de toutes nos méthodes de prévision (compte tenu des fonctions de répartition des écarts de $S$ et $S^{\prime}$ ), nous en avons sélectionné six à savoir :

- l'extrapolation linéaire;

- les différences premières d'ordre deux; 
- Muskingum avec extrapolation amont et sans prise en compte explicite de la pluie;

- Muskingum avec extrapolation amont et avec prise en compte explicite de la pluie;

- régression avec un point amont et sans prise en compte explicite de la pluie;

- régression avec un point amont et avec prise en compte explicite de la pluie.

La non prise en compte des méthodes à liaison spatiale avec correction, malgré l'amélioration qu'elles apportent dans les phases ascendantes et descendantes des hydrogrammes, vient du fait d'une forte surestimation des valeurs maximales; par rapport aux méthodes sans correction.

A partir de ces six méthodes nous avons construit cinq modèles qui se particularisent suivant l'une ou l'autre des deux approches précédentes.

- suivant l'approche (a) de compétition totale (modèles M1, M2).

- suivant l'approche (b) de compétition-association (modèles M3, M4, M5).

Pour juger de la qualité des modèles de compétition nous allons les confronter aux méthodes qui nous ont précédemment donné les meilleurs résultats, et avec lesquelles ils sont construits.

Pour ce faire nous représentons pour le délai de prévision de 6 heures seulement quelques graphes pour ne pas surcharger les figures:

a) Les fonctions de répartition des écarts $S$ des deux modèles confrontées à celles de la méthode de régression avec un point amont sans prise en compte explicite de la pluie (meilleure méthode au sens de $S$ ), (figure 3 ).

b) les fonctions de répartition des écarts $S^{\prime}$ des deux modèles confrontées à celles de la méthode de Muskingum avec extrapolation amont sans prise en compte explicite de la pluie (meilleure méthode au sens de $S^{\prime}$ ), (figure 4).

Lors de la construction des modèles M3, M4 et M5 nous n'avons pas utilisé l'extrapolation linéaire qui a le désavantage de surestimer systématiquement le débit maximal. Le souci majeur qui nous a guidé dans la fabrication de ces modèles a été de réduire l'erreur $S^{\prime}$ tout en gardant une valeur modérée de l'erreur $S$.

Au vu des résultats, les conclusions peuvent paraître surprenantes.

La compétition pure et dure des méthodes n'apporte pas de mieux sensible pour $S$ et peut provoquer une détérioration pour $S^{\prime}$. Ces résultats décevants peuvent peut-être s'expliquer par le fait que le micro-ordinateur change de méthode trop tard après avoir entériné une supériorité passée qui ne se maintient pas pour l'avenir donc pour la prévision.

\section{Le système manuel expert}

Le lecteur aura peut-être été déjà surpris de la simplicité voire de la rusticité des algorithmes que nous avons utilisés par rapport à la richesse et aux promesses des modèles sophistiqués parfois proposés dans la littérature scientifique hydrologique. Notre propos était délibéré, nous voulions apprécier le mieux apporté par la complexité pour ne pas dire la complication des modèles. Arrivés à cette étape de notre expérience, toujours tentés par la contestation iconoclaste nous nous sommes posés la question: quelles seraient les performances des prévisionnistes aux mains nues? Là est en définitive la justification de l'intrusion de l'informatique dans la prévision, faire mieux que le prévisionniste expérimenté.

Fouiller dans les archives des Services de prévision des crues est dans nos objectifs. Mais ceci constitue un travail fragmenté et de longue haleine. Pour gagner du temps, l'un de nous (C. THIRRIOT) a imaginé d'organiser des séances de simulation de crues avec des groupes de personnes de bonne volonté ayant des expériences diverses.

Le premier groupe est constitué d'ingénieurs suivant les enseignements de la Section spéciale hydraulique à l'ENSEEIHT auxquels s'étaient associés quelques chercheurs de l'Institut de mécanique des fluides.

Le deuxième groupe était l'équipe des prévisionnistes de Montauban. Evidemment les deux groupes n'étaient pas dans les mêmes conditions de compétence.

Le premier groupe avait de bonnes notions d'hydraulique mais aucune expérience de la prévision.

Le deuxième groupe connaissait la rivière, l'Aveyron, sur laquelle allait être tentée l'expérience.

Cette expérience pour certains tenait du canular et quelques uns par prudence rationnelle ont refusé d'y participer. Et nous saurions trop remercier tous ceux qui avec bonne humeur et application se sont prêtés à ce jeu du meilleur prévisionniste.

Nous n'insisterons pas sur la préparation du concours ni sur son déroulement à Toulouse ou à Montauban. Ceci est décrit dans un autre rapport (THIRRIOT et BERNEDE 1987). Pour ne pas trop allonger les séances de prévision nous avions choisi trois séquences successives : une en début de crue, la deuxième juste avant le maximum et la troisième au cours de décrue sans-à-coups.

Au total, il a été proposé de faire les prévisions à 15 instants. la figure 5 donne un exemple de résultats pour

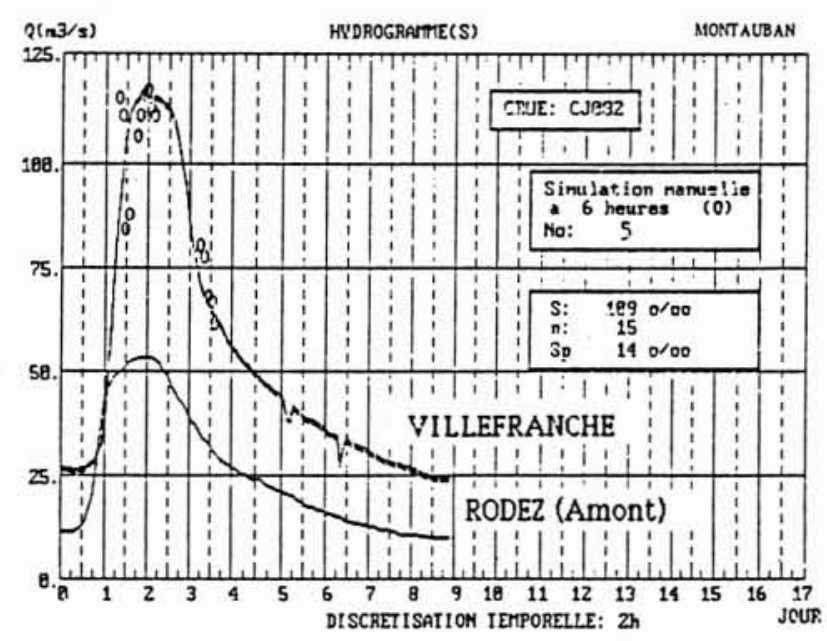

5. Hydrogrammes observées à Villefranche et Rodez. 
Fréquence cumulée

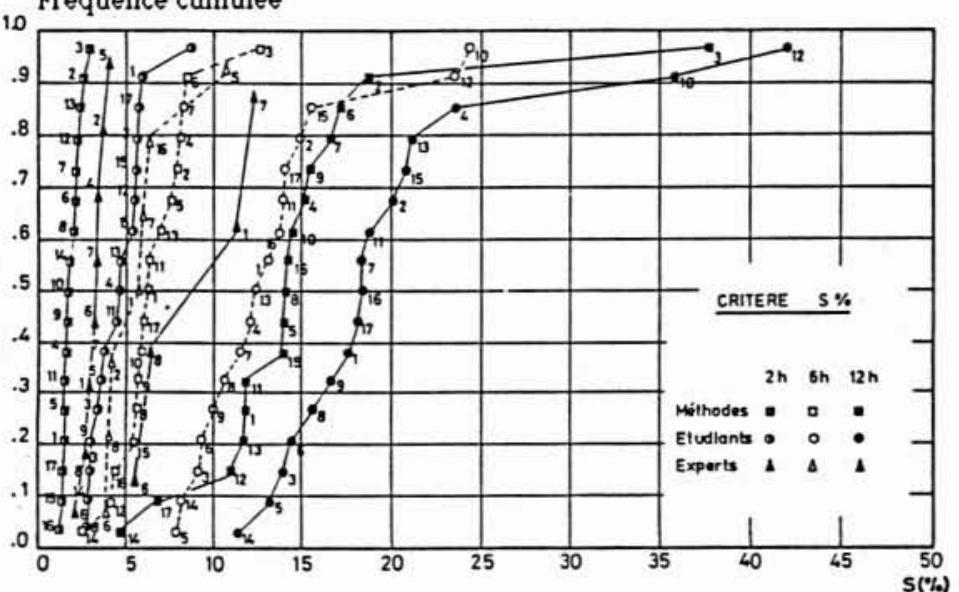

6. Fonctions de répartition des écarts type de prévision.
Fréquence cumulée

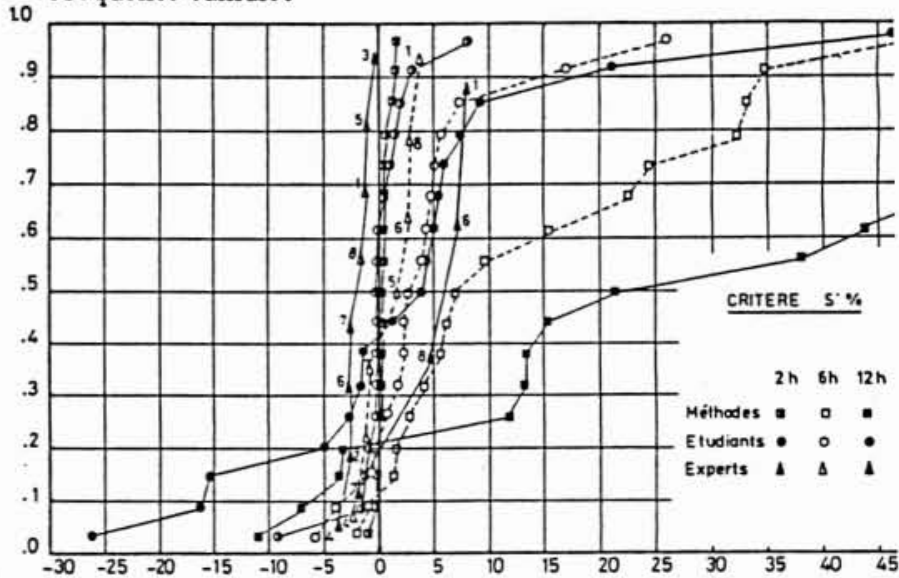

7. Fonction de répartition de l'erreur de prévision sur le maximum.
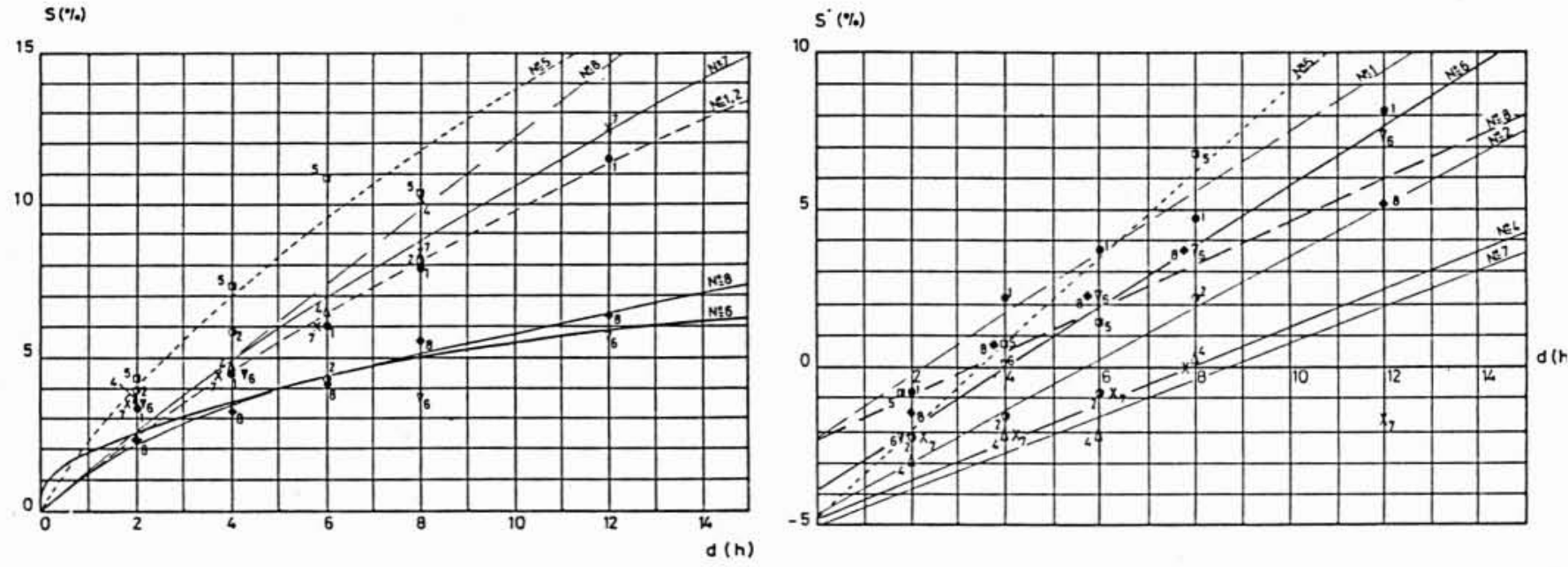

8 et 9. Graphes spécifiques des opérateurs (experts).

Etudiants

\begin{tabular}{|c|c|c|c|c|c|c|c|c|c|c|c|c|c|c|c|c|c|}
\hline $\begin{array}{c}x \\
\text { poctectr }\end{array}$ & 1 & $=$ & 3 & 4 & 5 & 6 & 7 & 8 & 9 & 10 & 11 & 12 & 13 & 14 & 15 & 16 & 17 \\
\hline$d^{*}$ & 0.06 & 0,10 & $0,45:$ & 0,43 & 0.48 & 0,43 & 0,37 & 0.44 & 0,49 & 0,13 & $0,1 ?$ & 0.23 & 0.33 & 0,25 & 0,11 & 0.12 & 0,07 \\
\hline$\alpha$ & 0.59 & $0.21 \mid$ & 0,01 & 0.01 & 0,06 & 0.06 & 0,04 & 0,03 & 0,02 & 0.04 & 0.12 & 0.01 & 0.03 & 0,25 & 0.19 & 0.21 & 0,41 \\
\hline$p$ & 0.96 & 0.95 & 0.97 & 0.99 & .098 & 0.97 & 0.99 & 0.95 & 0.98 & 0.98 & 0.94 & 0.99 & 0.99 & 0.95 & 0.96 & 0,95 & 0.93 \\
\hline
\end{tabular}

Experts

\begin{tabular}{|c|l|l|l|l|l|l|l|l|}
\hline $\mathrm{N}^{\circ}$ joveur & 1 & 2 & 3 & 4 & 5 & 6 & 7 & 8 \\
\hline$d^{*}$ & 0,78 & 0,74 & 0,36 & 0,32 & 0,41 & $-0,23$ & 0,84 & 0,49 \\
\hline$\alpha$ & 0,03 & 0,04 & 0,23 & $-0,01$ & 0,06 & $-1,72$ & 0,01 & 0,42 \\
\hline$\rho$ & 0,99 & 0,75 & 0,99 & 0,96 & 0,91 & 0,76 & 0,99 & 0,98 \\
\hline
\end{tabular}

Tableaux 2 et 3. - Paramétres de la relation $S(d)$ respectivement pour les étudiants et les experts.
Erutiant:

\begin{tabular}{|c|c|c|c|c|c|c|c|c|c|c|c|c|c|c|c|c|c|}
\hline $\begin{array}{c}N \\
\text { poctects }\end{array}$ & 1 & 2 & 3 & 4 & 5 & 6 & 7 & 8 & 9 & 10 & 11 & 12 & 13 & 14 & 15 & 16 & 17 \\
\hline$d^{*}$ & -3.7 & 13.6 & 1.3 & 0.5 & -0.4 & 2,2 & 1.2 & 1.5 & -0.7 & $-0,2$ & 0,05 & -0.2 & -0.6 & 2.2 & 0.2 & 8.2 & 1.8 \\
\hline$s_{0}^{\prime}$ & -0.7 & 1.3 & $-0,9$ & 3.8 & $-16,5$ & 0.5 & 1.7 & 1.8 & $-6,0$ & -25.0 & 119 & -30.0 & $-4,0$ & 1.0 & 6.2 & 0.3 & \\
\hline$p^{\circ}$ & 0.95 & 0.83 & 0.95 & & 0,20 & 0.94 & 0.97 & 0.99 & & -0.5 & 0,02 & $-0,3$ & & 0.97 & 0.98 & 0.91 & \\
\hline
\end{tabular}

Experts:

\begin{tabular}{|l|l|l|l|l|l|l|l|l|}
\hline $\mathrm{N}^{\circ}$ joveur & 1 & 2 & 3 & 4 & 5 & 6 & 7 & 8 \\
\hline$d^{* *}$ & 1,0 & 1,2 & 0,5 & 1,7 & 0,7 & 1,0 & 1,7 & 1,5 \\
\hline$S_{0}$ & 2,1 & 4,6 & 3,7 & 4,8 & 4,9 & 3,9 & 5,0 & 2,4 \\
\hline$p$ & 0,97 & 0,93 & 0,99 & 0,99 & 0,92 & 0,99 & 0,43 & 0,97 \\
\hline
\end{tabular}

Tableaux 4 et 5. - Parametres de la relation $S^{\prime}(d)$ respectivement pour les étudiants et les experts. 
un délai proche du temps de propagation. Du point de vue proximité observations et prévisions, au premier coup d'œil ce graphe soutient assez bien la comparaison avec les figures fournies par un traitement informatique et rencontrées dans les publications scientifiques.

Pour porter globalement un jugement rien de tel que les fonctions de répartition des erreurs relatives $S$ et $S^{\prime}$.

Pour donner une idée de la comparaison des résultats manuels avec les produits informatiques nous avons utilisé 17 méthodes. Mais il faut bien remarquer que la fonction de répartition informatique fait ainsi intervenir une batteries d'algorithmes dont on a pourtant éliminé la plupart à la suite de l'étude systématique réalisée par $H$. Habaieb dans sa thèse.

Chaque méthode est en quelques sorte comme un joueur. Mais la comparaison est un peu biaisée dès le départ car en réalité devant un cas nouveau on aurait hésité seulement entre quelques algorithmes (six au maximum).

Pour la comparaison, par souci d'homogénéïté, tout est ramené aux erreurs sur les débits (bien qu'à Montauban le jeu ait porté sur les hauteurs).

Les figures 6 et 7 montrent la comparaison des écarts relatifs pour quelques délais de prévision $(2,6$ et 12 heures). Les graphiques sont éloquents et rendent hommage à l'expérience des experts mais les résultats obtenus par les étudiants ne sont pas non plus caricaturaux.

Les méthodes informatiques résistent bien à la concurrence de l'homme pour les délais courts (jusqu'à 6 heures, proche du temps de propagation sur le bief) mais elles n'ont pas la prémonition des experts pour l'aventure audacieuse qu'est la prévision à 12 heures.

Il y a là en germe tout ce que nous comptons exploiter dans l'expérience globale et le savoir faire intuitif contenu dans le système manuel expert pour l'intégrer dans le système informatique expert.

Et à ce propos nous avons essayé de déceler les indices de comportement des opérateurs.

Tout de même un peu décontenancés par le volume et la disparité des résultats, nous avons cherché à cerner " l'équation personnelle " de chaque opérateur - comme on le dit de l'observateur. Aussi une première approche a consisté tout bonnement à construire les graphes des écarts relatifs en fonction du délai de prévision pour chacun des joueurs. (Figures 8 et g). Avaricieusement nous avons conservé même les résultats les moins bons au sens de la prévision pour essayer de juger statistiquement d'un autre critère 'qui est celui de la cohérence interne à chaque joueur.

Quelle que soit la qualité de la prévision du joueur la régularité des graphes $S(d)$ et $S^{\prime}(d)$ est étonnamment bonne.

Nous avons choisi de caractériser la cohérence interne par le coefficient de corrélation $\rho$ afférent au lissage des écarts $S$ par une parabole telle que

$$
d / d^{*}=S+\alpha S^{2}
$$

Les tableaux 2 et 3 montrent que pour la grande majorité des joueurs, l'indice de cohérence $\rho$ est très bon.

Les graphes concernant l'erreur sur le maximum $S^{\prime}(d)$ sont quasi linéaires pour la plupart des opérateurs et peuvent être lissés par une relation du type :

$$
d / d^{\star *}=S^{\prime}+S_{0}^{\prime}
$$

Cette fois, les valeurs de $S^{\prime}, S_{0}$ et $d^{\star \star}$ peuvent être négatives.

Un lissage a fourni les valeurs caractéristiques et l'indice de cohérence $\rho$ ' indiqués dans les tableaux 4 et 5 .

Deux particularités nous paraissent importantes:

- la valeur de $d$ pour laquelle l'erreur s'annule;

- le signe et l'importance de la pente du graphe (caractérisé par $d^{\star *}$ ).

De manière assez générale, pour la plupart des opérateurs, il y a sous estimation du maximum à bref délai et surestimation à fort délai (ce qui va assez bien dans le sens de la sécurité). Sur l'ensemble des 25 joueurs, un seul à eu un comportement très cohérent avec une tendance inverse en fonction du délai.

\section{Corrélation entre rapidité de réponse et qualité des prévisions (fig. 10 et 11).}

Dans l'examen des performances des joueurs nous nous sommes aussi posé la question de l'incidence de la durée de la réflexion, car le temps mis par les joueurs pour remplir une ligne de prévision à cinq délais variait beaucoup de l'un à l'autre.

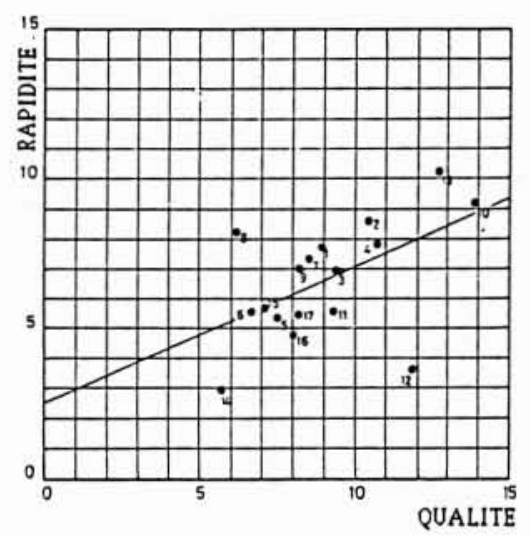

10. Graphe global qualité - rapidité du groupe étudiant.

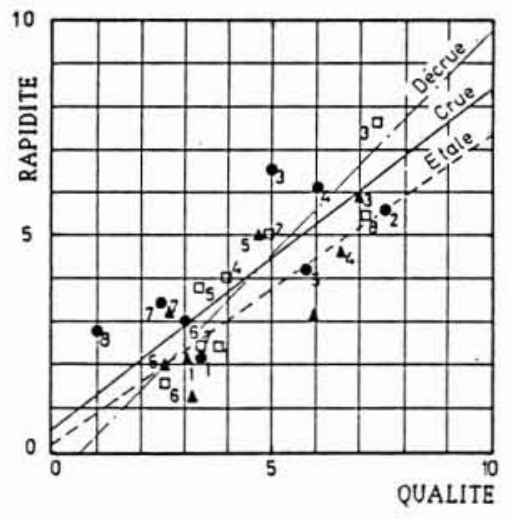

11. Graphes locaux qualité - rapidité du groupe expert. 
Comme tout de même il ne s'agit que d'un jeu, nous avons cherché à utiliser des critères simples ordinaux constitués par les rangs pondérés de classement pour la qualité et pour la rapidité de réponse (la pondération a permis de priviligier soit la prévision à court terme délais courts - soit les pronostics à long terme).

Les figures 10 et 11 donnent les exemples de résultats.

La disposition des joueurs dans le plan qualité/rapidité est à peu près la même qu'il s'agisse du court terme ou du long terme. Bien sûr, il ne faut pas extrapoler en disant que la réponse instantanée serait de meilleure qualité mais tels quels les résultats semblent suggérer que le premier mouvement est le bon.
A ce propos, il resterait encore à parler de la tactique des joueurs à maintenir leurs prévisions antérieures ou la façon de les corriger mais cela nous entraînerait trop loin.

Il faut bien finir par une autocritique, le jeu a été entrepris pour démythifier l'autorité de l'ordinateur sur la nature mais chemin faisant les réflexes rationalistes de l'intellectuel scientifique ont introduit une gestion automatisée des pronostics, dont les résultats paraîtront trop particuliers et trop fragmentaires pour en induire une hypothèse générale. Alors bien volontiers nous nous accusons de ce péché d'induction abusive et nous attendons le verdict d'autres expériences contestataires.

\section{Conclusion}

A l'issue de ce grand concours de "Victoire sur les crues ", la conclusion est humaniste et réconfortante : en définitive l'homme aux mains nues ne fait pas si mal que ça face à l'énorme appareil informatique. Nous nous en doutions un peu avant d'entreprendre l'opération et cela nous confirme dans la modestie de nos prétentions à encager la nature dans un moule rigide même sophistiqué.

D'une manière intuitive et synthétique, nous pensons même que d'utiliser beaucoup plus d'information n'arrangera pas beaucoup les choses. Encore une fois c'est le charme secret de l'entropie qui captée sous la forme de quantité d'information réapparait sous forme de critère de désordre thermodynamique.

Mais le profit de l'opération n'est pas uniquement négatif : elle suggère en particulier d'assurer la symbiose entre l'expérience des experts, leur mode d'appréhension synthétique et la rigueur du traitement informatisé.

Chemin faisant, nous avons vu qu'il y avait spécialisation dans l'exploit, le gagnant n'est pas le même pour l'écart-type et pour l'erreur sur le maximum (aussi bien du côté des méthodes que des hommes). Aussi dans l'exploitation du panier SME nous suggérons d'introduire l'unité de compte nouvelle : l'E.C.U. l'écart corrigé unifié qui ferait par exemple intervenir une pondération rationnelle (à partir des équations d'opérateur) à correction sélective suivant qu'il s'agit de montée, d'étale ou de décrue. Cette péréquation d'avis d'experts est d'ailleurs fréquente en économie.

Même chose tentée d'ailleurs pour l'exploitation des méthodes informatiques comme nous l'avons fait par le panachage et la compétition des algorithmes.

Enfin cette expérience ludique est une des premières étapes (peut être non orthodoxe) de l'élaboration d'un système expert informatique qui à la fois profiterait de l'expérience des experts et validerait l'apprentissage des prévisionnistes.

\author{
Adresse des auteurs \\ Messieurs C. Thirriot \\ J.P. Bernède \\ H. Habaieb \\ Laboratoire de l'ENSEEIHT \\ 2, rue Charles Camichel \\ 31071 Toulouse Cedex \\ Tél. : (16) 61.58.82.00 \\ et \\ Laboratoire de Banlève \\ Avenue du professeur Emile Soula \\ 31400 Toulouse \\ Tél. : (16) 61.28.58.00
}




\section{Bibliographie}

ArTKEN A.P., 1973. - Assessing systematic errors in rainfall run off models. J. Hydrol. Amsterdam. 20(2). pp. 121-136.

BACHET N., 1934. - Note sur la propagation et l'annonce de crue. Annales des Ponts et Chaussées. Mai-Juin 1934 - pp. 409-465.

Bocquillon C., Masson J.M., 1980. - Mise au point d'un modèle d'annonce de crue. Application à la basse Seine. Rapport LHM/10/80. U.S.T.L. Montpellier.

Box G.E.P., Jenkins G., 1970. - Times Series Analysis Forecasting and Control. Holden day San Francisco.

Chow K.C.A., WATT W.E., WATT D.G., 1983. - A stochastic dynamic model for real time flood forecasting. Water $R e$ sources Research. Vol. 19. $\mathrm{N}^{\circ} 3$, pp. 746-752.

De MARsily G., 1971. - La relation pluie-débit sur le bassin versant expérimental de l'Hallue. Rapport LHM/R/71/75. Laboratoire d'Hydrogéologie Mathématique. E.N.S. des Mines de Paris.

DUBREUIL P., 1974. - Initiation à l'analyse hydrologique. O.R.S.T.O.M.

E.G.C.E., 1967. - Bassin de l'Aveyron. Inventaire des sites de barrage de retenue. Tome 1. Délégation de l'Aménagement du Territoire et de l'Action Régionale. Mission Technique de l'Eau. Adour et Garonne.

Guillot P., Duband D., 1980. - Fonction de transfert pluiedébit sur les bassins versants de l'ordre de $1000 \mathrm{~km}^{2}$. La Houille Blanche $\mathrm{N}^{\circ} 4$. Avril 1980 - pp. 279-290.

HABAIEB H., 1987. - Contribution à l'étude critique des algorithmes de prévision de crue en temps réel. Exemple de l'Aveyron et de la Medjerdah. Thèse présentée à l'I.N.P. de Toulouse en vue d'obtenir le titre de Docteur de l'I.N.P.T.

Kitanidis P.K., Bras R.L., 1980. - Real time forecasting with a conceptual hydrologic mode. I : Analysis of uncertaintly II : applications and results. Water Ressources Research. Vol. 16. $\mathrm{N}^{\circ} 6$, pp. $1025-1044$.

LeBosse A. 1986. - Traitement des erreurs et procédures dégradées. EDF - LNH Chatou. Stage de prévision des crues. Toulouse 20-23 janvier.

Michel C., 1985. - Hydrologie appliquée aux petits bassins ruraux. C.E.M.A.G.R.E.G. - Section Hydrologie Hydraulique pluviale et souterraine. Antony. Octobre 1985.

MIQUEL J., 1984. - La prévision des crues : méthodes, calage et traitement des erreurs. Rapport EDF. Laboratoire National d'Hydraulique de Chatou.

NAGY A.S., Todini E., Wood, 1977. - A state space model for real time forecasting of hydrological time series. Journal of hydrological Sciences. 4(1), pp.61-76.

Nemec J., 1986. - Hydrological Forecasting: Design and
Operation of Hydrological Forecasting Systems. D. Reidel Publishing Company. $239 \mathrm{P}$.

O'CONNEL P.E., Clarke R.T., 1981. - Adaptative hydrological forecasting - a review. Hydrological Sciences. 26, 2, 6/1981, pp. 179-205.

O'DONNEL T., 1985. - A discret three parameter Muskingum procedure incorporating lateral inflow. Hydrological Sciences Journal 304. 12/1985, pp. 479-496.

ROche M., 1963. - Hydrologie de surface. ORSTOM.

Roche P.A., TAMIN R., 1985. - Analyse des procédures de décision en temps réel pour la prévision des crues. C.E.R.G.R.E.N.E. Juin.

Roche P.A., Torterotot J.P., 1987. - Prévision des crues sur la haute Garonne, utilisation d'une procédure multi-modèle. Rapport d'avancement $\mathrm{N}^{\circ} 1$. Méthodologie et critique des données. CERGRENE. Réf. DPP/SE-AE/JL - NL Nº 3156 , juin.

TAMIN R.Z., 1986. - Procédure de décision multi-modèles applicables à la prévision des crues en temps réel. Thèse présentée à l'Ecole Nationale des Ponts et Chaussées en vue d'obtenir le grade de Docteur-Ingénieur en Sciences et Techniques de l'Environnement.

Thirriot C., 1960. - Contribution à l'étude des régimes variés et des régimes transitoires dans les canaux d'usine. Thèse de Doctorat es Sciences physiques. Toulouse. $\mathrm{N}^{\circ}$ d'ordre 1262.

THIRrIOT C., 1968. - Comparaison des méthodes de calcul de la propagation des ondes de crues. S.H.F. - Dixième Journée de l'Hydraulique. La prévision des crues et la protection contre les inondations. Question II. rapport 10.

THIRriot C., 1984. - Une expérience coopérative : l'étude des crues et sécheresses sous l'égide de l'Institut Méditerranéen de l'Eau. Cinquième Conférence Internationale sur la Planification et la Gestion des Eaux. L'Eau en l'an 2000. Thème 3. Athènes. 1-4 octobre.

THIRRIOT C., BERNEDE J.P., 1987. - Doit-on vraiment sortir du S.M.E.? C.T. 718. Décembre.

Versiani B., 1983. - Modélisation de la relation pluie-débit pour la prévision des crues. Etude comparative de deux méthodes globales et application au bassin Gardon à Anduze. Thèse de Docteur-Ingénieur présentée à l'Université Scientifique et Médicale de Grenoble.

VIDAL J.J., 1979. - Validité de divers modèles de propagation des crues. Mémoire présenté en vue d'obtenir le Diplôme d'Ingénieur C.N.A.M.

VIDAL J.J., 1986. - Mise en œuvre d'une Méthode de propagation. méthode de Bachet, présentation et construction. S.H.C. - Stage "Prévision des crues". Toulouse, 20-23 janvier 1986. 